\title{
Torsion of a pedunculated subserous leiomyoma: a case report of a rare cause of acute abdominal pain in a pregnant woman
}

\author{
Marta D. L. Costa $^{1 *}$ (1) and Teresa Margarida Cunha ${ }^{2}$
}

\begin{abstract}
Background: Acute onset abdominal pain through the pedunculated leiomyoma torsion is a rather uncommon presentation and a rare source of acute abdominal pain in the emergency department during pregnancy. Due to its rarity, this condition is not always considered, leading sometimes to inappropriate or unnecessary therapeutic approaches. Given that there are no specific clinical findings, radiological examination represents a fundamental tool for achieving the correct diagnosis. Pelvic ultrasound is usually the first imaging approach, but MRI is recommended for further evaluation, showing high diagnostic accuracy.
\end{abstract}

Case presentation: A 35-year-old primigravid woman presented at the gynecological emergency department at 21 weeks of gestation, with a complaint of sudden onset of strong intensity right abdominal pain. Vital signs and blood test results were normal. A pelvic ultrasound was requested and showed a heterogeneous pelvic mass in the right lower quadrant, between the uterine body and the right ovary (which was not enlarged and presented normal venous and arterial blood flow) raising the suspicion of a right ovary tumor or an exophytic uterine tumor. An MRI was performed and revealed a well-defined and heterogeneous mass, with predominantly high signal intensity on T2-weighted images (WI) with and without fat saturation and with hyperintense areas on axial T1Wl; the presence of bridging vessels in the interface between the tumor and the uterus was also demonstrated-the "bridging vessel sign." The tumor presented restricted diffusion with high signal intensity on axial diffusionweighted imaging (b1000) and low signal intensity on the axial ADC map. The set of MR imaging features was consistent with the torsion of a pedunculated subserosal uterine leiomyoma with hemorrhagic necrosis. A successful conservative approach was the first-option treatment, with no further surgical intervention.

Conclusion: The torsion of a pedunculated subserous leiomyoma should be considered in a pregnant woman with acute onset abdominal pain. The correct diagnosis, based on clinical and radiological findings, is essential for good outcomes concerning maternal and fetal well-being, allowing the safest and most appropriate treatment. A conservative approach seems to be a safe and appropriate first-option treatment to this condition.

Keywords: Leiomyoma, Torsion, Abdominal pain, Pregnancy, Case report

* Correspondence: martacosta.med@gmail.com

'Department of Radiology, Centro Hospitalar Universitário de Lisboa Central,

R. Beneficência 8, 1050-099 Lisbon, Portugal

Full list of author information is available at the end of the article 


\section{Background}

Despite uterine leiomyomas represent the most common gynecologic tumors with a prevalence estimated in $20-40 \%$ [1], the estimated incidence rate in pregnancy is rare, ranging from 0.1 to $3.9 \%$ [2]. Most of them remain asymptomatic; nevertheless, complications may occur, although rare, such as torsion of pedunculated subserous leiomyoma. The diagnosis is based on clinical and radiologic examination. Patients usually present with acute onset abdominal pain, but there are no specific clinical features. Ultrasound (US) is usually the first imaging modality in the evaluation of this entity; however, its sensitivity is operator-dependent; for better and further evaluation, magnetic resonance imaging (MRI) is recommended, showing high diagnostic accuracy. There is no treatment consensus of uterine leiomyomas in pregnancy, and each patient must be evaluated individually. However, surgical intervention should not be performed routinely in all cases due to the high risk of hemorrhagic or obstetric complications.

\section{Case presentation}

A primigravid woman, 35 years old, 21 weeks of gestation, was admitted at the gynecological emergency department with a complaint of sudden onset of strong intensity right lower quadrant abdominal pain. Vital signs were stable. Physical examination revealed tenderness to deep palpation in the right lower quadrant of the abdomen, without guarding. Blood test results were normal. She had no relevant medical or family history. No relevant past interventions were reported.

A transabdominal pelvic US was requested and showed a viable, single fetus; a heterogenous pelvic mass was found in the right lower quadrant, between the uterine body and the right ovary, which was not enlarged and presented normal venous and arterial blood flow. These findings did not support the hypothesis of ovarian torsion, raising the suspicion of a right ovary tumor or an exophytic uterine tumor. To rule out renal colic, a kidney US was also performed, which showed no hydroureteronephrosis. (The images were obtained with the ultrasound available at the gynecological emergency department from a peripheral hospital, which has no connection with the picture archiving and communication system, so the US examinations were not recorded and their access was lost.)

The patient was hospitalized in need of pain management with intravenous analgesics. After 1 week, she was discharged and referred to an oncology center for better investigation, where an MRI was performed within 2 weeks later (about 3 weeks after the onset of symptoms). MRI revealed a well-defined and heterogeneous mass, with predominantly high signal intensity on T2-weighted images without (Fig. 1a, b) and with (Fig. 1c, d) fat saturation and with hyperintense areas on axial T1-weighted images consistent with the presence of blood content (Fig. 1e). It was also demonstrated the presence of bridging vessels in the interface between a subserosal leiomyoma and the uterus on T2-weighted images without and with fat saturation (Fig. 1) - the "bridging vessel sign" [3] which revealed the uterine origin of the pelvic mass. Besides that, both ovaries were identified on axial T2weighted images, without any lesions (Fig. 2). The tumor presented restricted diffusion with high signal intensity on axial diffusion-weighted imaging (b1000) (Fig. 3a) and low signal intensity on axial ADC map (Fig. 3b), as usual in the presence of blood content. The set of MR imaging features was consistent with the torsion of a pedunculated subserosal uterine leiomyoma with hemorrhagic necrosis.

A conservative approach was decided with symptomatic therapy. Subsequent follow-up with pelvic US was performed every 3 weeks; the tumor had progressively smaller dimensions, which corroborated the MRI diagnosis. (As the patient decided to perform serial follow-up US examinations with her gynecologist in a private practice, unfortunately, the images were not provided). The pregnancy continued its full normal course, without any complications, with a normal vaginal birth at 40 weeks.

\section{Discussion}

The reported prevalence of uterine leiomyomas throughout pregnancy is rare, ranging from 1.6 to $10.7 \%$, depending upon the trimester of assessment (first versus second) and the size threshold for reporting the tumor (e.g., varies between $\geq 0.5$ and $>3 \mathrm{~cm}$ ) [4].

Chronic pain is the main reported symptom; however, most of them remain asymptomatic.

Acute onset abdominal pain through the pedunculated leiomyoma torsion, as our patient experienced, is a rather uncommon presentation and a rare source of acute abdominal pain in the emergency department during pregnancy [5]. The uterine size in pregnancy and the uterine involution in the postpartum raise the risk of torsion [6]. The thin and long pedicle allows greater motility of the leiomyoma, increasing the possibility of torsion. Enlarged leiomyoma is also associated with irreversible torsion, but the cutoff size is not defined [7].

The diagnosis is based on clinical and radiologic examination. There are no specific clinical signs or symptoms associated with this condition. The US is usually the first imaging modality in the evaluation of uterine leiomyomas; it can help in identifying a pedunculated subserosal leiomyoma and checking the ovaries' integrity [8]. Sometimes, color Doppler may demonstrate a twisted pedicle with blood flow absence within the tumor, suggesting leiomyoma torsion; however, the twisted pedicle is often thin and hardly seen [9].

As we know, US sensitivity is operator-dependent and subjective to interpretive error, and as occur in our case, a pedunculated leiomyoma may be mistaken with an adnexal mass, especially if the stalk is not visualized. 

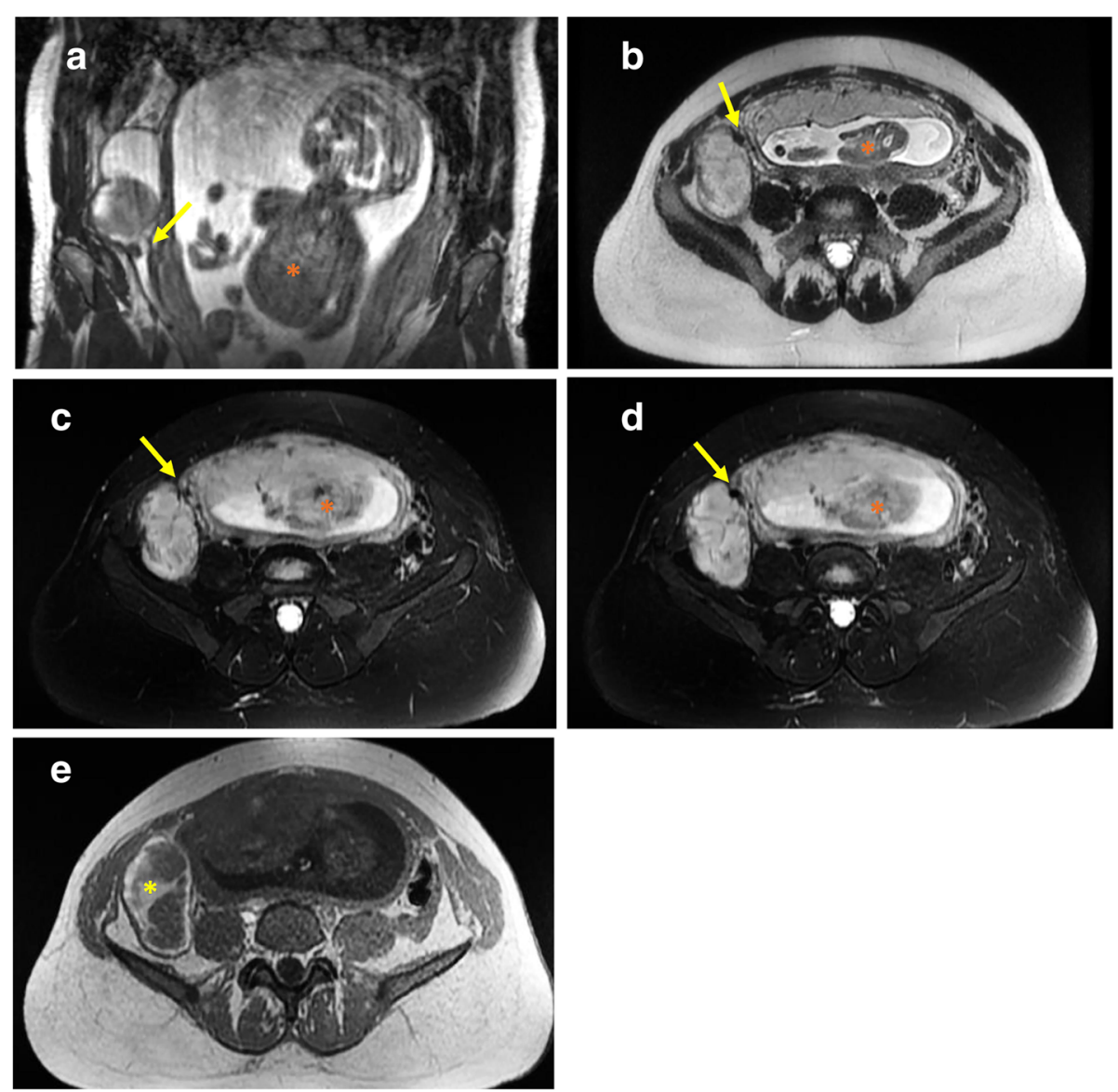

Fig. $1 \mathrm{MRI}$ shows a well-defined and heterogeneous tumor, with predominantly high signal intensity on coronal (a) and axial T2-weighted images without (b) and with (c, d) fat saturation images; it contains hyperintense areas on axial T1-weighted images (e) consistent with the presence of blood content (yellow asterisk). It also demonstrated a hypointense vascular pedicle representing feeding vessels that arise from the uterine arteries, coursing from the uterus into an adjacent exophytic pelvic mass (yellow arrow) — "bridging vessel sign"; this sign reveals the uterine origin of the pelvic mass. A single fetus is also seen (orange asterisk)

MRI is the most accurate imaging technique for detection, characterization, and localization of pelvic tumors, usually recommended for further evaluation [10].

Typical uterine leiomyomas on MRI usually show hypointense homogeneous signal T1- and T2-weighted images. However, the pedicle twisting of a pedunculated subserous leiomyoma leads to subsequent ischemia, necrosis, and gangrene. So, the leiomyoma shows a heterogeneous high signal intensity on T2-weighted images and an intermediate to high signal intensity on T1-weighted images related to hemorrhagic necrosis or ischemia, respectively. Peripheral low signal intensity on T2-weighted images and high signal intensity on T1-weighted images are consistent with venous flow obstruction [7].

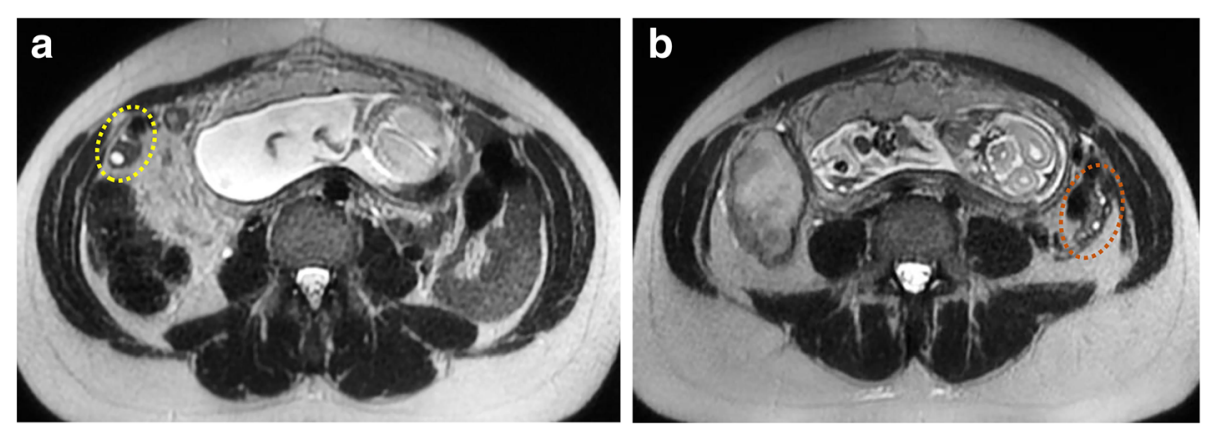

Fig. 2 Both ovaries are identified on axial T2-weighted images (right-yellow ellipse in a; left-orange ellipse in b) 

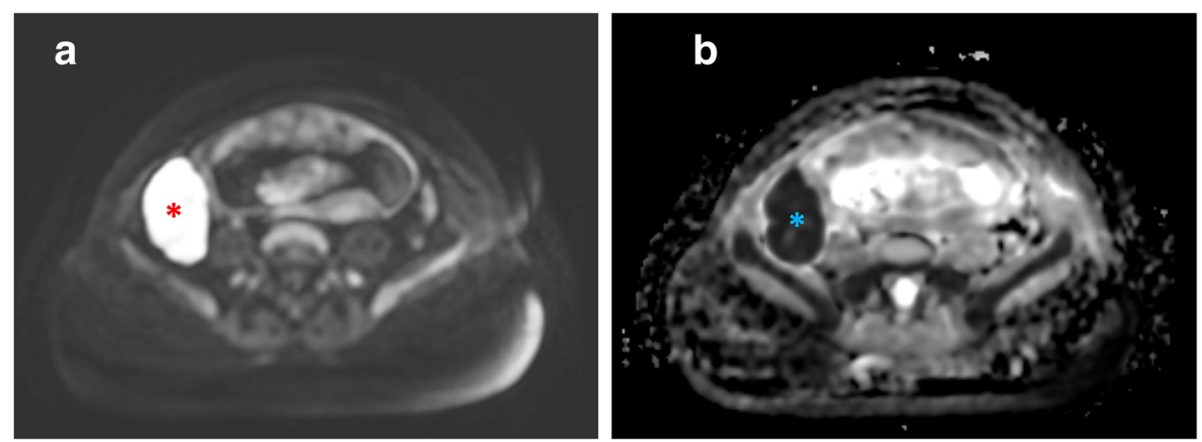

Fig. 3 The tumor presents restricted diffusion with high signal intensity on axial diffusion-weighted imaging (b1000) (red asterisk in a) and low signal intensity on axial ADC map (blue asterisk in $\mathbf{b}$ ). This is consistent with the presence of blood content, as identified on T1-weighted images

The "bridging vessel sign" refers to the presence of bridging vessels in the interface between a subserosal leiomyoma and the uterus [3]; this reveals the uterine origin of the pelvic mass, helping to differentiate between a pedunculated subserosal uterine leiomyoma from other peri-uterine masses of ovarian, adnexal, or bowel origin. It is also important to identify both ovaries.

There is no treatment consensus of uterine leiomyomas in pregnancy [7]. Each patient must be evaluated individually, and treatment should be promptly decided for good outcomes concerning maternal and fetal wellbeing. During pregnancy, a conservative approach with symptomatic therapy may be considered as the firstoption treatment to the torsion of a pedunculated leiomyoma, safely and with a good prognosis, as occur in our case. This condition is a rare surgical emergency [8], and due to the high risk of hemorrhagic or obstetric complications (miscarriage, preterm delivery, risk of uncontrolled hemorrhage leading to hysterectomy), myomectomy should not be performed routinely in all cases. However, if severe pain persists after pharmacologic treatment for more than $72 \mathrm{~h}$, surgical intervention should be the next therapeutic approach [11].

\section{Conclusion}

The torsion of a pedunculated subserous leiomyoma is a rare complication of uterine leiomyoma in pregnancy but should be considered in a pregnant woman with acute onset abdominal pain. The correct diagnosis, based on clinical and radiological findings, is essential for good outcomes concerning maternal and fetal well-being, allowing the safest and most appropriate treatment. A conservative approach seems to be a safe and appropriate first-option treatment to this condition.

\section{Abbreviations}

MRI: Magnetic resonance imaging; US: Ultrasound

\section{Acknowledgements}

Not applicable.

\section{Authors' contributions}

MDLC reviewed the available literature and drafted the manuscript. TMC provided clinical information and medical images, as well as critically revised the manuscript. Both authors read and approved the final version of the manuscript.

\section{Funding}

The authors declare that there were no external sources of study for the performance of this article.

\section{Availability of data and materials Not applicable.}

Ethics approval and consent to participate Ethical approval is not required for the publication of isolated case reports. The patient was informed about the procedure, and a written informed consent was obtained.

\section{Consent for publication}

Written informed consent was obtained from the patient for publication of this case report and accompanying images.

\section{Competing interests}

All authors declare no competing interests.

\section{Author details}

${ }^{1}$ Department of Radiology, Centro Hospitalar Universitário de Lisboa Central, R. Beneficência 8, 1050-099 Lisbon, Portugal. 'Department of Radiology, Instituto Português de Oncologia de Lisboa Francisco Gentil, R. Prof. Lima Basto, 1099-023 Lisbon, Portugal.

Received: 29 April 2020 Accepted: 27 July 2020

Published online: 05 August 2020

\section{References}

1. Vitale SG, Tropea A, Rossetti D, Carnelli M, Cianci A (2013) Management of uterine leiomyomas in pregnancy: review of literature. Updates Surg 65(3): 179-182

2. Basso A, Catalano MR, Loverro G, Nocera S, Naro ED, Loverro M et al (2017) Uterine fibroid torsion during pregnancy: a case of laparotomic myomectomy at 18 weeks' gestation with systematic review of the literature. Case Rep Obstet Gynecol 2017:4970802

3. Dionisio T, Gomes FV, Cunha TM (2014) Non-ovarian pelvic masses: key concepts and useful clues to the radiologist. Eur Congr Radiol C-1191

4. Ouyang DW, Norwitz ER (2019) Uterine fibroids (leiomyomas): issues in pregnancy. UpToDate.

5. Larson NP, Bridwell RE, Cibrario A et al (2019) Necrotic fibroid mimicking ovarian torsion in a second trimester of pregnancy. Cureus. 11(11):e6172 
6. Mickel I, Bollmann R, Chaoui R, Lau HU (1995) Torsion of the myoma pedicle as a rare cause of ileus in puerperium. Geburtshilfe Frauenheilkd 55(12):721-723

7. Laia Y-L, Chen Y-L, Chen C-A, Cheng W-F (2018) Torsion of pedunculated subserous uterine leiomyoma: a rare complication of a common disease. Taiwan J Obstet Gynecol 57(2):300-303

8. Gaye YFO, Niang MM, Gassama O, Thiam O, Thiam M, Cisse CT (2017) The torsion of a pedunculated uterine leiomyoma, a rare etiology of acute pelvic pain: about an observation and a review of the literature. Gyneco Womens Health Res 2017:1(1)

9. Kim H-G, Song YJ, Na YJ, Choi O-H (2013) A case of torsion of a subserosal leiomyoma. J Menopausal Med 19(3):147-150. https://doi.org/10.6118/jmm. 2013.19.3.147

10. Khan AT, Shehmar M, Gupta JK (2014) Uterine fibroids: current perspectives. Int J Women's Health 6:95-114

11. Yasa C, Dural O, Demiral I, Comba C, Demir O, Bastu E (2016) Torsion of a pedunculated subserous leiomyoma in a pregnant woman: a rare case report. Int Surg 101

\section{Publisher's Note}

Springer Nature remains neutral with regard to jurisdictional claims in published maps and institutional affiliations.

\section{Submit your manuscript to a SpringerOpen ${ }^{\circ}$ journal and benefit from:}

- Convenient online submission

- Rigorous peer review

- Open access: articles freely available online

High visibility within the field

- Retaining the copyright to your article

Submit your next manuscript at $\boldsymbol{\nabla}$ springeropen.com 